\title{
Cryopiece, a novel carrier with faster cooling rate, high recovery rate and retrieval rate, for individual sperm cryopreservation
}

\author{
Zi-Jue Zhu ${ }^{1,2 \#}$, Jing Zhai ${ }^{1,2 \#}$, Jian-Lin $\mathrm{Hu}^{3}$, Yi-Zhou Wang ${ }^{1,2}$, Wei Chen ${ }^{3}$, Feng Liu ${ }^{1,2}$, Yong-Hua Huang ${ }^{4}$, \\ Zheng $\mathrm{Li}^{1,2}$ \\ ${ }^{1}$ Department of Andrology, Urological Medical Center, Shanghai General Hospital, Shanghai Jiao Tong University School of Medicine, Shanghai, \\ China; ${ }^{2}$ Shanghai Key Lab of Reproductive Medicine, Shanghai Jiao Tong University, Shanghai, China; ${ }^{3}$ Department Of ART, Shanghai General \\ Hospital, Shanghai Jiao Tong University School of Medicine, Shanghai, China; ${ }^{4}$ Institute of Refrigeration and Cryogenics, School of Mechanical \\ Engineering, Shanghai Jiao Tong University, Shanghai, China \\ Contributions: (I) Conception and design: ZJ Zhu, Z Li; (II) Administrative support: Z Li; (III) Provision of study materials or patients: JL Hu, W \\ Chen, F Liu, YH Huang; (IV) Collection and assembly of data: ZJ Zhu, J Zhai, YZ Wang, YH Huang; (V) Data analysis and interpretation: ZJ Zhu, \\ J Zhai; (VI) Manuscript writing: All authors; (VII) Final approval of manuscript: All authors. \\ \#These authors contributed equally to this work. \\ Correspondence to: Prof. Zheng Li. Department of Andrology, Urologic Medical Center, Shanghai General Hospital, Shanghai Jiao Tong University \\ School of Medicine, 86 Wujin Road, Shanghai 200080, China. Email: Lizhengboshi@sjtu.edu.cn.
}

Background: Cryopreservation of extremely few spermatozoa is still a major challenge for male fertility preservation. This study aims to evaluate the cooling rate, recovery rate, and retrieval rate, along with other parameters of spermatozoa that cryopreserved using Cryopiece, a novel carrier, for individual sperm cryopreservation.

Methods: Semen samples from 60 fertile donors were collected, and each semen sample was screened for motile sperm and mixed with cryoprotective agent (CPA), and then frozen using Cryopiece, micro-straw, and mini-straws. The cooling rate, retrieval rate, and recovery rate, morphology, DNA fragmentation index (DFI) and mitochondrial membrane potential (MMP), were compared among the un-frozen sperm and the sperm cryopreserved using these carriers.

Results: Cryopiece possessed the fastest cooling rate. After freeze-thaw, the average retrieval rate of sperm cryopreserved using Cryopiece was $96.25 \%$, and the average recovery rate was $64.40 \%$, which were higher than that of sperm cryopreserved using the other two carriers $(71.42 \%$ and $54.30 \%$ for micro-straw, and $63.54 \%$ and $58.04 \%$ for mini-straw, respectively). There was no significant impact on DFI after sperm cryopreservation, and no significant difference in morphology between sperm cryopreserved using these carriers was observed. Though MMP of sperm changed significantly after cryopreservation, micro-straw maintained sperm MMP better than Cryopiece and mini-straw did, while no significant difference was observed in MMP between sperm cryopreserved using Cryopiece and mini-straw.

Conclusions: Cryopiece produced satisfying retrieval and recovery rates in sperm cryopreservation and should be an ideal carrier for cryopreservation of small number of sperm.

Keywords: Individual sperm cryopreservation; recovery and retrieval rate; DNA fragmentation index (DFI); morphology; mitochondrial membrane potential (MMP)

Submitted Jul 11, 2020. Accepted for publication Jan 08, 2021.

doi: $10.21037 /$ tau-20-1080

View this article at: http://dx.doi.org/10.21037/tau-20-1080 


\section{Introduction}

Due to the deterioration of the environment, unhealthy lifestyle, and the genetic factors, more and more couples are suffering from infertility (1). According to the data released by the WHO (World Health Organization), $10-15 \%$ couples were infertile and about $50 \%$ of them were associated with male factors. Today, majority of infertile couples will be successfully treated by assisted reproduction technology (ART), especially intracytoplasmic sperm injection (ICSI) for severe male factors such as oligospermia or asthenozoospermia $(2,3)$. The pregnancy rate is about $40-45 \%$ for ICSI cycle (4). Unfortunately, in some cases, ICSI cycle may be canceled due to no sperm available in semen or testicular biopsy specimens (5). Therefore, before commencing ICSI cycle, it is very important to cryopreserve small number of sperm retrieved from semen of severe oligospermic men or testicular biopsy sample from non-obstructive azoospermia (NOA) men.

Cryopreservation of small number of sperm is very challenging with conventional cryopreservation carriers, which could cause loss and seriously injury of sperm (6). Several carriers have been developed to improve the outcome of sperm cryopreservation. A micro-straw has been reported to be useful carrier for sperm density $<2 \times 10^{6} / \mathrm{mL}$ (7). Nevertheless, to those who suffer from cryptozoospermia or NOA, the number of retrieved sperm is usually below 100 . Micro-straw is incompetent to preserve such few sperm.

In literatures, many innovated carriers for individual sperm cryopreservation were reported, such as Cryoloop, Cryotop, and Cell sleeper (8-12). Even empty zona pellucida was reported to be employed (13). Artificial synthetic hollow hyaluronan microcapsules and agarose capsules were also be developed for sperm cryopreservation (14-16). However, these methods are still not been widely used in ART clinics.

A novel carrier called Cryopiece, which was suitable for cryopreservation of extremely few sperm, had been developed previously (17). During sperm cryopreservation using Cryopiece, micromanipulation was used to load sperm onto specific carrier individually. No additional transfer of sperm during the freeze-thaw procedure, which could ensure almost no loss of sperm during following process.

Though there had been successful delivery of health baby with the help of Cryopiece for extremely few spermatozoa cryopreservation (17), a systemic evaluation of Cryopiece has not yet been conducted. The aim of this study was to compare the sperm retrieval and recovery rates, and impact on sperm DFI, morphology, and mitochondrial membrane potential (MMP) between Cryopiece, micro- or mini-straws for cryopreservation of small number of sperm. We present the following article in accordance with the TREND reporting checklist (available at http://dx.doi.org/10.21037/ tau-20-1080).

\section{Methods}

\section{Sperm sample and preparation}

The study was conducted in accordance with the Declaration of Helsinki (as revised in 2013). The study was approved by the Institutional Review Board of Shanghai General Hospital (license number of ethics statement: 2016KY196). Informed consents were signed by all sperm donors. Semen samples of 60 healthy donors (ages ranged from 23 to 29 years, mean, $25.3 \pm 3.4$ years) were randomly collected from Department of Andrology, Urological Medical Center, Shanghai General Hospital, Shanghai Jiao Tong University. Semen was obtained by masturbation after abstinence for 2-7 days. Then, a routine semen analysis was performed according to the WHO Laboratory Manual for the Examination and Processing of Human Semen $\left(5^{\text {th }}\right.$ Edition), and parameters of enrolled semen samples were within the following range: volume of semen $>2 \mathrm{~mL}$, sperm concentration $>20 \times 10^{6} / \mathrm{mL}$, and progressive motility rate (PR) $>60 \%$.

Because of that only the motile sperm would be chosen during individual sperm cryopreservation, to make a comparison with other types of carrier, motile sperm should be used in all cryopreservation procedure. A modified swim-up method was employed for motile sperm screening. Briefly, about 1-2 mL liquefied semen was centrifuged at $600 \mathrm{~g}$ for $10 \mathrm{~min}$, remove the upper semen plasma and retain about $200 \mu \mathrm{L}$ liquid. Re-suspend the precipitate and add the suspension at the bottom of a clean $1.5 \mathrm{~mL}$ tube, then about $1 \mathrm{~mL}$ mHTF (Modified HTF Medium, Ref.90126, Irvine Scientific, USA) supplied with 5\% HSA (human serum albumin) was carefully covered on the suspension. The tube was incubated in a $37^{\circ} \mathrm{C}$ incubator for at least 1 hour, and then the upper mHTF (about 500-700 $\mu \mathrm{L}$ to avoid the inclusion of unscreened immotile sperm) was collected. The screened sperm were then counted under microscopy. Samples with a sperm concentration over $2 \times 10^{6} / \mathrm{mL}$ (with the total sperm number over $1 \times 10^{6}$, which would be sufficient for the following examination), and motile sperm above $95 \%$ after swim-up screening were used in following examinations. The screened sperm derived 
from the same semen specimen were divided into different groups for treating and detecting in all following works, sperm derived from different semen specimens were served as biological repeats.

\section{Three types of carrier: Cryopiece, micro-, and mini-straws}

Cryopiece is a novel carrier designed for individual sperm cryopreservation. It is a thin slice made from polystyrene, about $1 \times 3 \mathrm{~cm}$ in size, and TC (tissue culture) treated. On the end of Cryopiece, there exists a handle made from $3 \mathrm{M}$ tape, which enable the operator to move it with the help of tweezers, without touching the surface of Cryopiece (Figure 1A). It could be put in a vial for preservation. Before using, Cryopiece was sterilized via ethylene oxide treatment. Recently, a kind of improved Cryopiece was developed (Figure 1B). The slice is fixed to an injection molded handle, which could be mounted on an improved vial cap tightly. The cap could be screwed onto the vial with the Cryopiece mounted, thus avoiding the rocking of the Cryopiece inside the vial during transferring and cryopreservation (Figure 1C,D,E). In present work, the improved Cryopiece was utilized.

Micro-straws which had been described in our previous work and commercially available mini-straws (Ref 010261, CryoBiosystem, Paris, France) that had been widely used in our clinical practice were used for comparison (7).

\section{Detection of cooling rate during cryopreservation}

In this study, a data acquisition unit PXle-1073 from National Instruments ${ }^{\circledR}$ was adopted to measure the real-time temperature during freezing and thawing at a high frequent (at $10 \mathrm{kHz}$ sample rate), along with a $\Phi 0.1 \mathrm{~mm}$ diameter T-type (copper-constantan) thermocouple as the sensor. The small volume of the sensor ensured the instant response to the temperature change of the sample. To provide the zerodegree reference temperature, an Omega ${ }^{\circledR}$ TRCIII-A icepoint Calibration Reference Chamber was used.

To measure the cooling rate of the cryoprotective agent (CPA) on the Cryopiece during freeze-thaw process, the T-type thermocouple was attached on the Cryopiece by adhesive tape to avoid drop-off of the probe at cryogenic temperature. The point was completely immerged in the CPA drops. Cryopiece was put in the vial and fumigated with vapors of liquid nitrogen at $5 \mathrm{~cm}$ above the liquidair interface of liquid nitrogen while the cooling rate was recorded. When the temperature reached $-130^{\circ} \mathrm{C}$, the vial was dropped into liquid nitrogen. For thawing, the Cryopiece was picked out of the vial, immersed into $37{ }^{\circ} \mathrm{C}$ pre-heated light mineral oil (Oil for Embryo Culture, Ref. 9305, Irvine Scientific, USA) immediately and incubated for $3 \mathrm{~min}$. The cooling rates of micro-straw and mini-straw loaded with CPA were also detected in the same way for making a comparison, the only difference of the performance was that micro-straw and mini-straw were put in a plastic holder instead of vial during fumigation, and the thawing of these straws was performed by $37^{\circ} \mathrm{C}$ water-bath for $3 \mathrm{~min}$.

\section{Sperm cryopreservation}

For cryopreservation using Cryopiece, swim-up screened sperm was loaded in Petri dish (Petri Dish, Ref. 1006, BD/ Falcon, USA) as micro strips.

CPA (Cryosperm, Ref. 10670010F, Origio, Denmark) was mixed with mHTF supplemented with $5 \%$ HSA at a ratio of 1:1, and loaded as drops on to Cryopiece, $2 \mu \mathrm{L}$ for each drop. Then Cryopiece was laid in the Petri dish.

Cover the micro strips and the Cryopiece with light mineral oil. ICSI needle (ICSI, Ref. MIC-35-35, Origio, USA) was used to transfer the motile sperm from the micro strips to the CPA drops individually (Figure $1 F$ ).

After transferring the spermatozoa, Cryopiece was picked out of the oil, drain it with tissue carefully and put it into vial. Then, the vial with Cryopiece was fumigated with vapor of liquid nitrogen at $5 \mathrm{~cm}$ above the liquid-air interface of liquid nitrogen for at least $10 \mathrm{~min}$ and stored in the liquid nitrogen finally.

For thawing, light mineral oil put in the Petri dish was pre-heated in incubator at $37{ }^{\circ} \mathrm{C}$, and then Cryopiece was picked out of the vial immediately and put into the warmed oil. The frozen drops would be thawed within seconds.

Cryopreservation and thawing of sperm specimen using micro and mini-straw was performed as described previously (7).

Briefly, as for micro-straw, several drops of $50 \mu \mathrm{L}$ mixed sperm specimen and CPA were placed into a culture dish. We pushed up the outer metal shell of micro-straw and exposed the inner tube to aspirate mixed sample with a sterile syringe, and then pushed down the outer metal shell to protect the pine end of the inner tube. Finally, microstraw was put in the holder and fumigated with vapor of liquid nitrogen for at least $10 \mathrm{~min}$ before being transferred into liquid nitrogen. For thawing of sperm, the microstraws were put in $37{ }^{\circ} \mathrm{C}$ incubator immediately after picked out from the liquid nitrogen, and incubated for $3 \mathrm{~min}$. The 

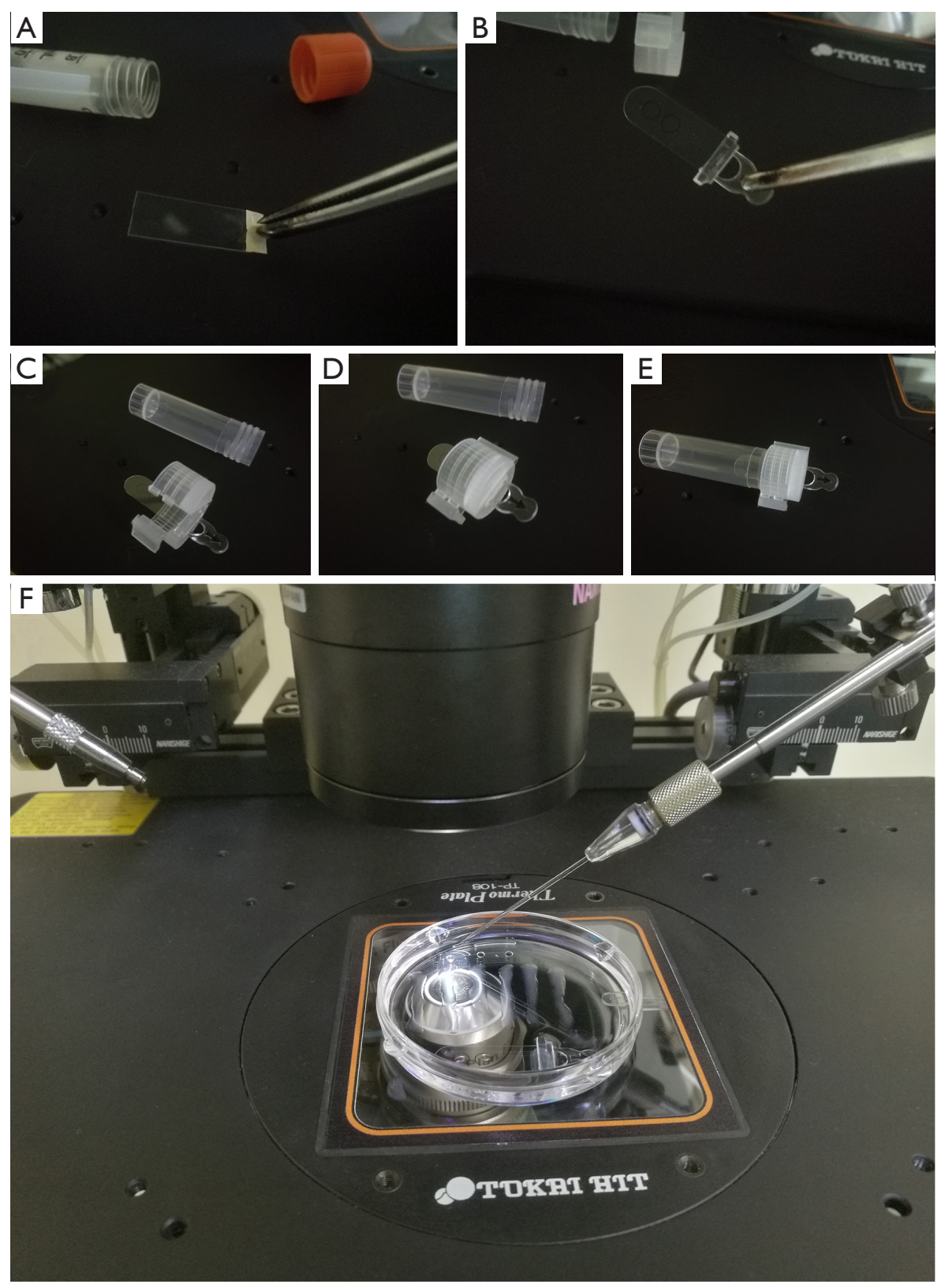

Figure 1 Cryopiece and the application of it in individual sperm cryopreservation. (A) The original version of Cryopiece was a thin slice made from polystyrene. On one end of Cryopiece, there exists a handle made from $3 \mathrm{M}$ tape, which enable the operator to move it with the help of tweezers, without touch the surface of Cryopiece. It could be put in a vial for preservation; (B) the appearance of the improved Cryopiece. The slice is fixed to an injection molded handle; $(\mathrm{C}, \mathrm{D}, \mathrm{E})$ the injection molded handle could be mounted on an improved vial cap tightly. The cap could be screwed onto the vial with the Cryopiece mounted, thus avoiding the rocking of the Cryopiece inside the vial during transferring and cryopreservation; (F) sperm specimen was loaded in Petri dish as micro strips. Cryoprotective agent (CPA) mixed with mHTF was loaded as drops on to Cryopiece. Then Cryopiece was laid in the Petri dish. Cover the micro strips and the Cryopiece with light mineral oil. Then, ICSI needle was used to transfer the motile sperm from the micro strips to the CPA drops on Cryopiece individually. ICSI, intracytoplasmic sperm injection. 
solution in the micro-straws was lightly blown into prewarmed recovery solution at $37^{\circ} \mathrm{C}$ in a $5 \% \mathrm{CO}_{2}$ incubator for $10 \mathrm{~min}$ before further examinations.

As for mini-straw, the mixture of sperm specimen and CPA was drawn into the mini-straws, and then the straws were sealed using a heating-sealing and then put in the holder, fumigated with vapor of liquid nitrogen for at least $10 \mathrm{~min}$ before being transferred into liquid nitrogen for preservation.

The straws were thawed in a water bath at $37^{\circ} \mathrm{C}$ for $3 \mathrm{~min}$. Then, we cut the sealed ends of the straws and lightly blew out the solution into pre-warmed recovery solution. The following steps were the same with that of the micro-straw.

\section{Recovery rate and retrieval rate calculation}

For testing and comparing the recovery rate and retrieval rate after cryopreservation and thawing using Cryopiece, sperm from 20 donors were processed through swim-up procedures. Each semen specimen was used for the calculation individually. A total of 20 spermatozoa were transferred into each CPA drop loaded on the Cryopiece through the micromanipulation. Then the Cryopiece was frozen and thawed as mentioned above. The recovery rate and retrieval rate were checked under an inverted microscope.

For micro-straw and mini-straw, the specimens were cryopreserved and thawed as described previously. The number of sperm in the sample and the number of motile sperm were checked respectively under microscope before freezing and after thawing. Motile sperm was defined as sperm with any motion, including vibration. The retrieval rate was calculated as the ratio of sperm count after thawing to before freezing. The recovery rate was defined as the ratio of motile sperm count after thawing to before freezing.

\section{DNA fragmentation index (DFI) examination}

For DFI examination, adequate spermatozoa were transferred into several CPA drops loaded on the Cryopiece. Then the Cryopiece was frozen and thawed as mentioned above. The drops were recovered carefully after thawing, avoiding the mixing of oil. Sperm chromatin dispersion (SCD) test using Halo-tech sperm DFI test kit (Halosperm, Ref. HT-HS1, Spain) was carried out to exam the DFI according to the instruction.

Briefly, sperm specimen was mixed with pre-melt lowmelting agarose and loaded onto the treated side of the slide provided in the kit, covering with a coverslip, being careful to avoid air bubbles. The slide was then put into a fridge at $4{ }^{\circ} \mathrm{C}$ for cooling. After the agarose was solidified, the coverslip was removed carefully, and the slide was immersed in the acid denaturant solution, incubating it for $7 \mathrm{~min}$. Then, lysis solution was used to incubate the slide for $25 \mathrm{~min}$. After being washed with water for $5 \mathrm{~min}$, the slide was dehydrated in ascending series of ethanol, and left to dry at room temperature. Wright stain was employed to stain the slide. Wright stain solution was mixed with phosphate buffer at a ratio of 1:1. The slide was kept in horizontal station, and drops of mixed Wright stain solution was added to cover the agarose layer, staining for about 2-5 min, and then the slide was washed briefly in tap water and air dry. A microscope was used to observe the staining result. The haloes appeared around the heads of sperm were counted. DFI value was defined as the ratio of sperm with small halo (the halo width is similar or smaller than $1 / 3$ of the minor diameter of the core) or without halo to that with bigger halo.

Un-frozen sperm specimen was used as control. A minimum of 300 sperm were counted for each sample before and after freeze-thaw procedure; 60 samples from the 60 donors were used for biological repeatability.

\section{Sperm morphology evaluation}

For evaluating the sperm morphology, screened sperm were divided into 5 groups. Fresh group: no CPA added, unfrozen; CPA added group: CPA added, un-frozen; Cryopiece group: CPA added, cryopreserved using Cryopiece; microstraw group: CPA added, cryopreserved using microstraw; mini-straw group: CPA added, cryopreserved using mini-straw. The freeze-thaw procedure was performed as described above. Smears were prepared using the recovered drops, un-frozen specimen served as control. Diff-Quik (sperm Morphology Quick-Staining Solution, Ref. BA4157B, Baso, China) was used to stain the smears. Briefly, sperm smears were immersed in Diff-Quik Fix for $5 \mathrm{~s}$ for fixation, and then immersed in Diff-Quik I and Diff-Quik II for $5 \mathrm{~s}$ in turn for staining. The stained smears were washed by fresh water and air dried. The normal morphology rate was evaluated according to the WHO laboratory semen examination manual 5 th edition. A total of 200 sperm were assessed for morphology in each sample. Sisty samples from the 60 donors were used for biological repeatability.

\section{Flow cytometric evaluation of MMP $(\Delta \psi m)$}

For evaluating the MMP of spermatozoon, JC-1 fluorescent dye (5,5',6,6'-tetrachloro-1-1',3,3' tetraethylbenzimidazolylc 


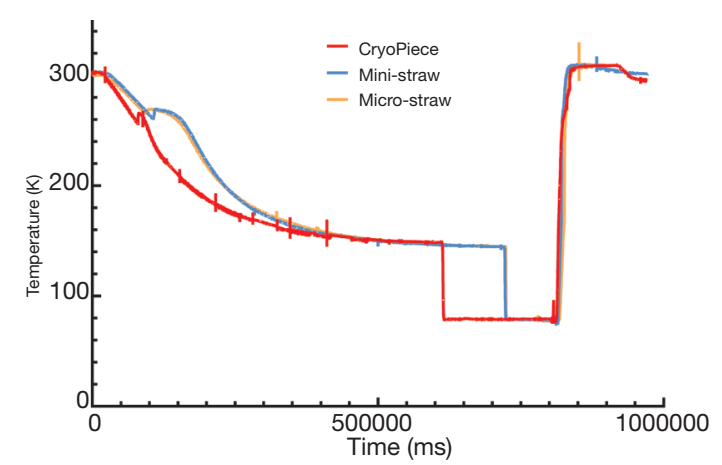

Figure 2 The cooling curves of the three types of carriers loaded with cryoprotective agent (CPA). The cooling rate of Cryopiece is significantly faster than that of micro-straw and mini-straw. Microstraw shares similar cooling curve with mini-straw.

arbocyanine iodide) (Yeasen, CAS3850-30, Shanghai, China) was used for monitoring and directly measuring the MMP $(\Delta \psi \mathrm{m})$, which is a good indicator of mitochondrial function. $\mathrm{JC}-1$ is a cationic lipophilic probe that can selectively enter and distinguish the mitochondria with low and high $\Delta \psi \mathrm{m}$, and has a dual emission. Red fluorescence (FL-3) corresponds to active and functional mitochondria, and green fluorescence (FL-1) corresponds to inactive or inoperative mitochondria. The MMP was defined as red/green ratio which could indicate the whole mitochondrial activity in sperm. The higher the MMP, the better the mitochondrial function was. All experiments were performed on FACS Calibur cytometer (BD Biosciences). A minimum of 10,000 sperm were calculated for MMP in each sample; 60 samples from the 60 donors were used for biological repeatability.

A control group was prepared by incubating the sperm with p-trifluoromethoxy carbonyl cyanide phenylhydrazone (CCCP), a mitochondrial uncoupling agent, for $20 \mathrm{~min}$ at room temperature. This uncoupler agent alters the red/green fluorescence decreasing the ratio value. Other 5 groups were the same as those in the sperm morphology evaluation.

\section{Statistical analysis}

The retrieval rate and recovery rate were calculated by comparing sperm numbers and motility rate after freeze-thaw procedure. The data of retrieval rate, recovery rate, sperm morphology, DFI, and MMP were analyzed by paired $t$-test or one-way of variance (ANOVA) using GraphPad Prism 8.2.1 software (GraphPad Software, Inc., La Jolla, CA, USA), $\mathrm{P}<0.05$ was considered to be statistically significant. If the $\mathrm{P}$ value was less than 0.05 by ANOVA, the Tukey Significant
Difference test was performed using the same program.

\section{Results}

\section{Cryopiece has the fastest cooling rate}

The cooling rate during sperm cryopreservation showed very difference between Cryopiece, micro-straw and mini-straw. The cooling rate of Cryopiece was obviously faster than micro-straw and mini-straw, showing 29.12, 20.99 , and $20.63{ }^{\circ} \mathrm{C} / \mathrm{min}$ from 27 to $0{ }^{\circ} \mathrm{C}, 44.20,26.05$, and $25.90{ }^{\circ} \mathrm{C} / \mathrm{min}$ from 0 to $-50^{\circ} \mathrm{C}, 43.53,39.06$, and $35.20{ }^{\circ} \mathrm{C} / \mathrm{min}$ from -50 to $-80{ }^{\circ} \mathrm{C}$, and $6.99,6.30$, and $6.27^{\circ} \mathrm{C} / \mathrm{min}$ from -80 to $-130^{\circ} \mathrm{C}$, respectively, thus rapidly get through the freezing point, during which the intracellular ice and extracellular ice formed (Figure 2, Table 1).

\section{The sperm cryopreserved using Cryopiece has the highest recovery rate and retrieval rate}

After thawing, the recovery rate and retrieval rate of the sperm were calculated. The average retrieval rate and recovery rate of sperm cryopreserved using Cryopiece were $96.25 \%$ and $64.40 \%$, respectively. For micro-straw, the average retrieval rate and recovery rate were $71.42 \%$ and $54.30 \%$, respectively. For mini-straw, the average retrieval rate and recovery rate were $63.54 \%$ and $58.04 \%$, respectively. The recovery rate and retrieval rate of sperm cryopreserved using Cryopiece was significantly higher than that of sperm cryopreserved using micro-straw and ministraw significantly (Figure 3).

\section{No significant difference was observed in DFI between sperm before cryopreservation and after cryopreservation using Cryopiece}

Semen provided by 60 donors was used to evaluate the DFI of sperm. The average DFI of sperm before and after cryopreservation using Cryopiece were $13.80 \%$ and $14.18 \%$, respectively. There was no significant difference in DFI between sperm before cryopreservation and after cryopreservation using Cryopiece (Figure 4).

\section{No significant difference was observed in normal morphology rate between sperm cryopreserved using the three types of carrier}

Normal sperm morphology rate was analyzed after thawing 
Table 1 Comparison of cooling rate during freezing (liquid nitrogen fumigation) and thawing between Cryopiece, micro-straw and mini-straw

\begin{tabular}{lccr}
\hline & & \multicolumn{2}{c}{ Cooling rate $\left({ }^{\circ} \mathrm{C} / \mathrm{min}\right)$} \\
\cline { 2 - 4 } Temperature range $\left({ }^{\circ} \mathrm{C}\right)$ & Cryopiece & Micro-straw & Mini-straw \\
\hline 27 to 0/freezing & 29.12 & 20.99 & 20.63 \\
0 to $-50 /$ freezing & 44.20 & 26.05 & 25.90 \\
-50 to $-80 /$ freezing & 43.53 & 39.06 & 35.20 \\
-80 to $-130 /$ freezing & 6.99 & 6.30 & 6.27 \\
-193 to 27 /thawing & 832.3 & 922.30 & 854.95 \\
\hline
\end{tabular}

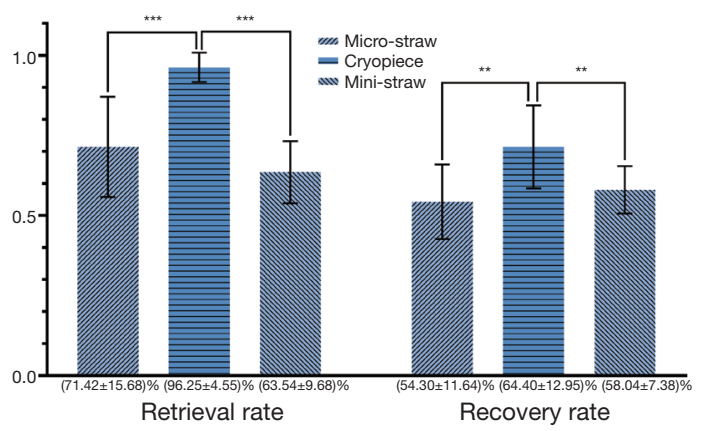

Figure 3 Comparison of the retrieval and recovery rates between sperm cryopreserved using the three types of carriers. The recovery rate and retrieval rate of sperm cryopreserved using Cryopiece were significantly higher than that of sperm cryopreserved using mini-straw and micro-straw. $\left.{ }^{* *}, \mathrm{P}<0.01 ;{ }^{* *}, \mathrm{P}<0.001\right)$.

according to the guidance of WHO laboratory semen examination manual 5 th edition. The average normal morphology rates of sperm were $24.20 \%$ in fresh group, $18.34 \%$ in CPA added group, $15.47 \%$ in Cryopiece group, $16.27 \%$ in micro-straw group, and $15.39 \%$ in mini-straw group, respectively. The normal morphology rate of CPA group was significantly decreased after adding CPA compared with fresh group $(\mathrm{P}<0.001)$. Besides, the normal morphology rates of cryopreserved sperm specimens were also significantly decreased. However, there was no significant difference between Cryopiece group, microstraw group, and mini-straw group (Figure 5).

\section{Micro-straw maintained MMP best while no significant difference was observed in MMP between sperm cryopreserved using Cryopiece and mini-straw}

The effect of the three types of carrier on MMP in human sperm was measured using JC-1 staining (Figure 6A).
Analysis by flow cytometry showed the average MMP of Cryopiece group and mini-straw group were 0.1143 and 0.1118 , respectively. No significant difference between these two groups was observed. The average MMP of microstraw group was 0.1345 , which was significantly higher than that of Cryopiece group and mini-straw group. Besides, it was found the average MMP of fresh group and CPA added group were 0.1370 and 0.1475 , respectively. The MMP of CPA added group was higher than that of fresh group significantly. The MMP of cryopreserved sperm decreased significantly compared with CPA added group. (Figure 6B).

\section{Discussion}

For a long time, clinical technicians of fertility preservation have been looking for an ideal carrier for the cryopreservation of small number of sperm. Such carrier should have an appropriate cooling rate to reduce the freezing injury and should not introduce additional damage on genetic materials of sperm. Furthermore, the carrier should minimize the loss of sperm during freezing and thawing procedure since each spermatozoon retrieved from patients with severe oligospermia and NOA is precious. These spermatozoa may be the last chance for them to father their own offspring through ICSI cycle. So, it was not allowed to use the spermatozoa retrieved from the patients for experimental study. Therefore, we use the spermatozoa donated by normal fertile men.

It was reported that the cooling rate of micro-straw, mini-straw, and $0.5 \mathrm{~mL}$ straw were $262.80,153.45$, and $42.99^{\circ} \mathrm{C} / \mathrm{min}$ from -50 to $-80{ }^{\circ} \mathrm{C}$, and $94.32,57.12$, and $12.71{ }^{\circ} \mathrm{C} / \mathrm{min}$ from -80 to $-130{ }^{\circ} \mathrm{C}$, respectively (7). However, these data were obtained via putting the carriers directly in the fumigation liquid nitrogen for analysis, which was different from the clinical protocol we following. In clinical work, before the specimen was fumigated, a cover 

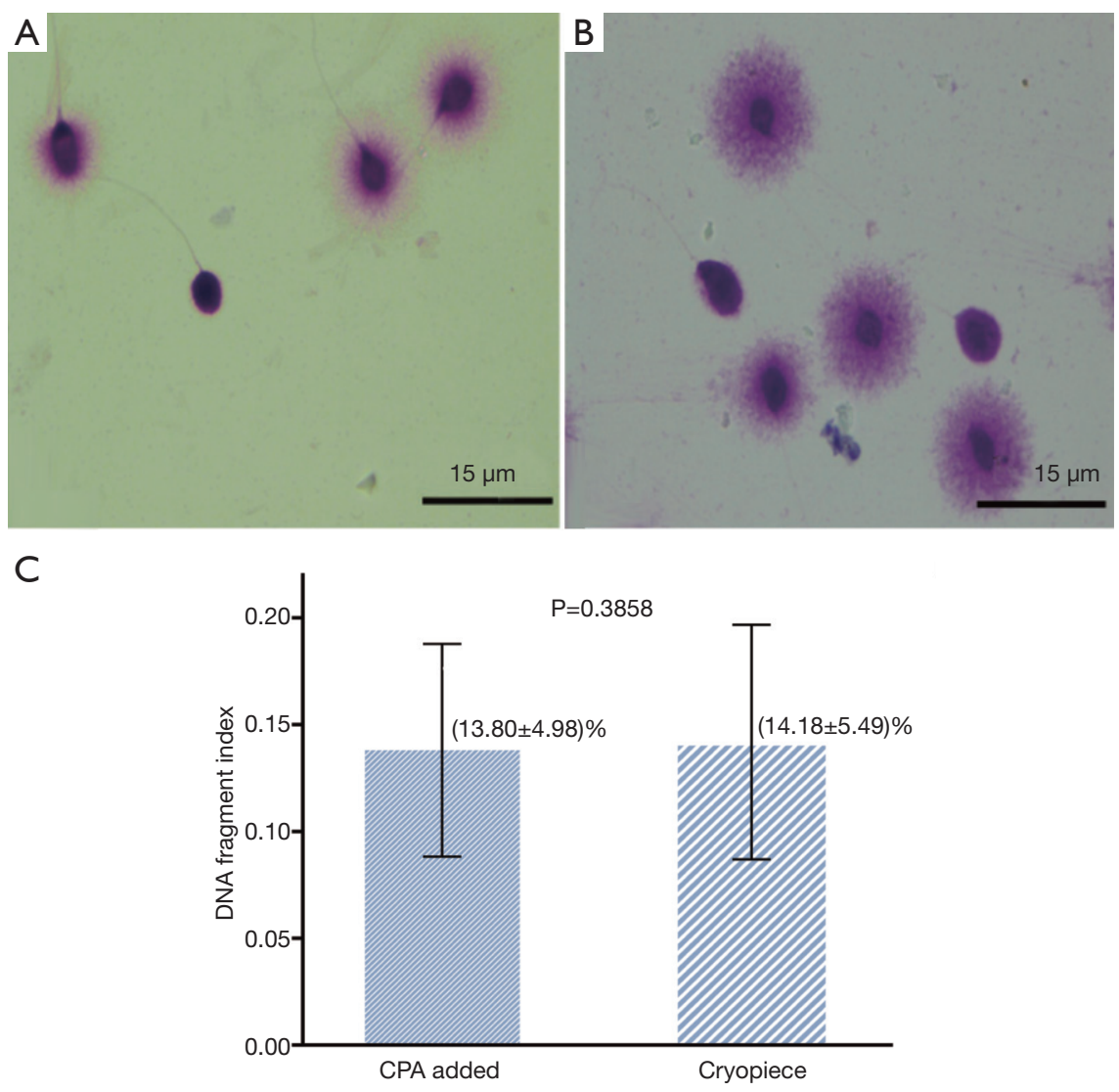

Figure 4 Sperm DNA fragmentation index (DFI) examination. Sperm chromatin dispersion (SCD) test was applied for DFI examination. Wright's stain was employed to stain the slides. SCD test generates a halo over the sperm head; the larger the halo was, the less DNA damage presented. (A) Typical picture of DFI examination results of fresh sperm; (B) typical picture of DFI examination results of sperm after cryopreservation using Cryopiece; (C) comparison of DFI values between the two groups. There was no significate difference between them.

held by aluminum frame was used to hold the carrier. So in current study, the cooling rate is lower than that detected in previous work, but closer to clinical situation.

Compared with that of the other carriers, the cooling rate of Cryopiece is faster. Since it was reported that sperm can maintain the integrity of DNA, chromatin, and acrosome better in a lower cooling rate rather than higher ones $(18,19)$, fumigation in nitrogen vapor above the liquid nitrogen was chosen as the method for sperm cryopreservation rather than direct immersion in liquid nitrogen. The faster cooling rate ensures the carrier could response more quickly to the change of environment temperature, getting through the crystalizing point more quickly, avoiding the formation of crystal, and reducing the cryo-injury to the sperm. So when fumigation was applied for sperm cryopreservation, Cryopiece would be a better carrier for reducing the cryoinjury to the sperm.
The average recovery rate of sperm cryopreserved using Cryopiece was $64.40 \%$, the average retrieval rate was $96.25 \%$, almost all the sperm transferred to the CPA drops can be found after thawing. In fact, there should be no loss of sperm occurred after cryopreservation using Cryopiece, since no container replacement took place during the operation. No sperm would be lost due to adhesion on the container as it happened when other carriers were used. However, some sperm would swim up to some height in the drops, and it would be hard to find them by adjusting the focal length, so actually not all the sperm could be found after thawing. We believe that the retrieval rate could be further improved by reducing the volume of CPA drops. Anyway, the recovery rate and retrieval rate, especially the retrieval rate, of sperm cryopreserved using Cryopiece were significantly higher than that using micro-straw or mini-straw, showing great advantage of Cryopiece in the 

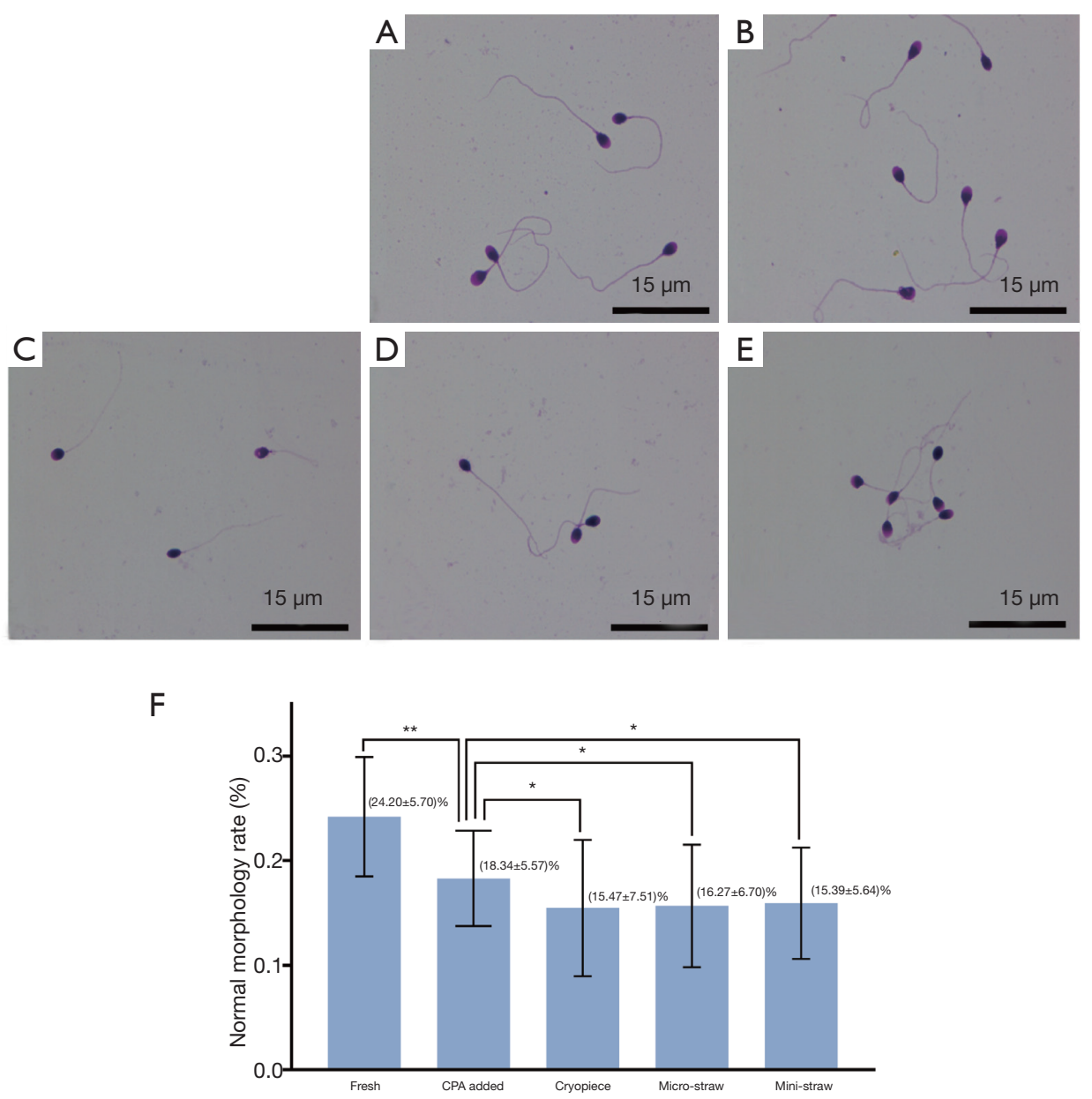

Figure 5 Sperm morphology analysis. Diff-Quik staining was employed to stain the sperm. (A) Typical morphology of fresh sperm; (B) typical morphology of sperm with cryoprotective agent (CPA) added; (C) typical morphology of sperm cryopreserved using Cryopiece; (D) typical morphology of sperm cryopreserved using micro-straw; (E) typical morphology of sperm cryopreserved using mini-straw. (F) Histogram shows significant difference in the normal morphology rate between the fresh sperm and frozen-thawed sperm. However, no significant difference exists in the normal morphology rate between sperm cryopreserved using Cryopiece and mini-straw or micro-straw. (*, $\left.\mathrm{P}<0.05 ;{ }^{* *}, \mathrm{P}<0.01\right)$.

application in extremely few sperm cryopreservation.

The cryopreservation using Cryopiece is safe to the genetic materials of sperm. DFI test showed that there was no significant difference observed in DNA integrity between fresh sperm and sperm cryopreserved using Cryopiece. The results indicated that no additional DNA damage was produced during cryopreservation using Cryopiece. DNA fragmentation could lead to embryo development stop and abortion $(20,21)$. In our previous work, it had been proved that cryopreservation of sperm using micro-straw or ministraw would not impair sperm DNA integrity (7). Although there were contradictory reports on the influence of sperm cryopreservation on DFI $(7,18)$, current work confirmed that it is safe for DNA to cryopreserve the sperm using Cryopiece.

The morphology analysis of the sperm cryopreserved using Cryopiece also proved to be satisfied. The head of the sperm would be injured by the formation of crystal during cryopreservation, and the tail of the sperm would be coiled due to the application of CPA and the change of osmotic pressure during liquid freezing. Though the normal morphology rate of the cryopreserved sperm was shown to be significantly decreased compared with that of fresh sperm, there was no significant difference between sperm cryopreserved using Cryopiece and mini-straw or microstraw. 
A

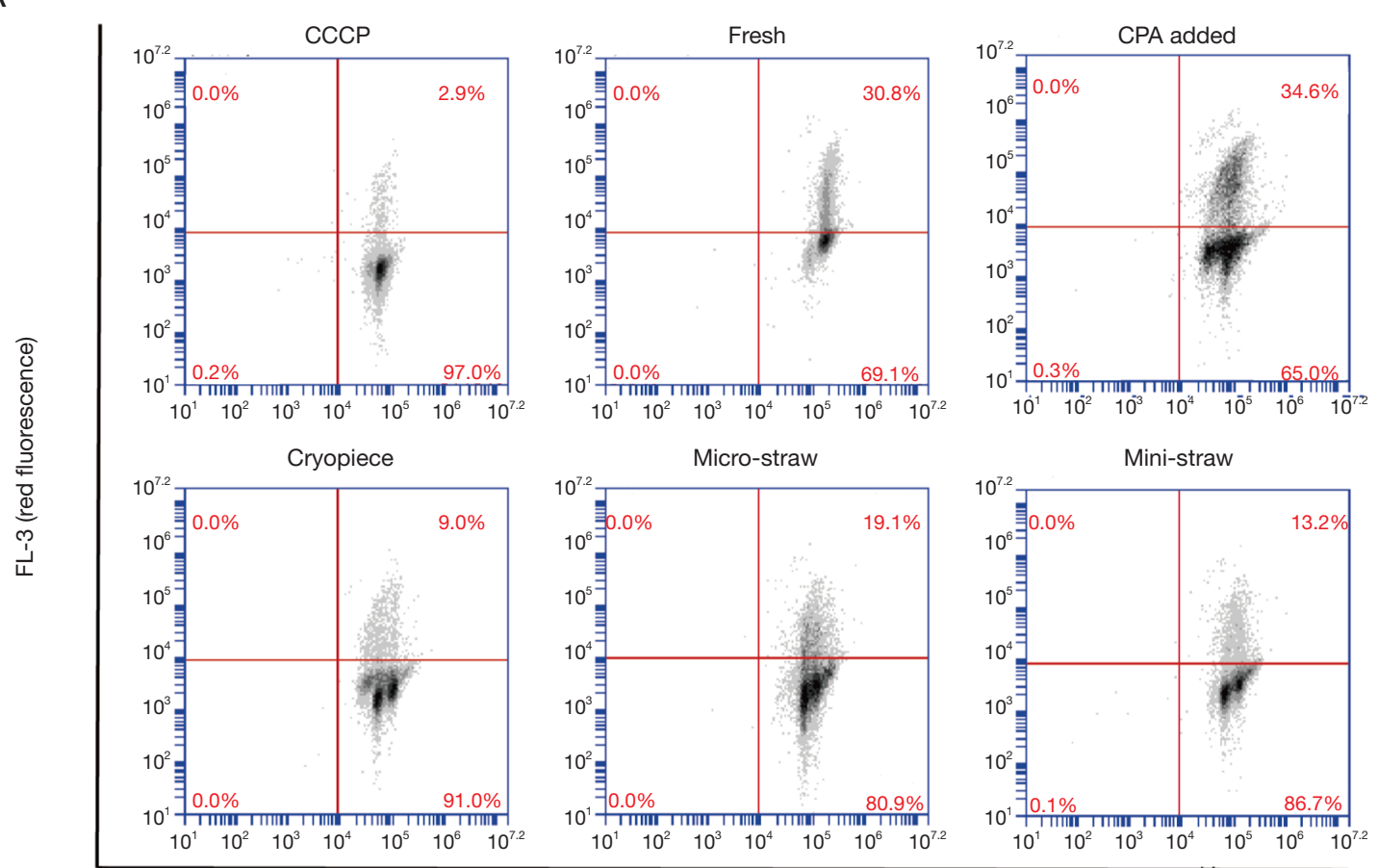

FL-1 (green fluorescence)

B

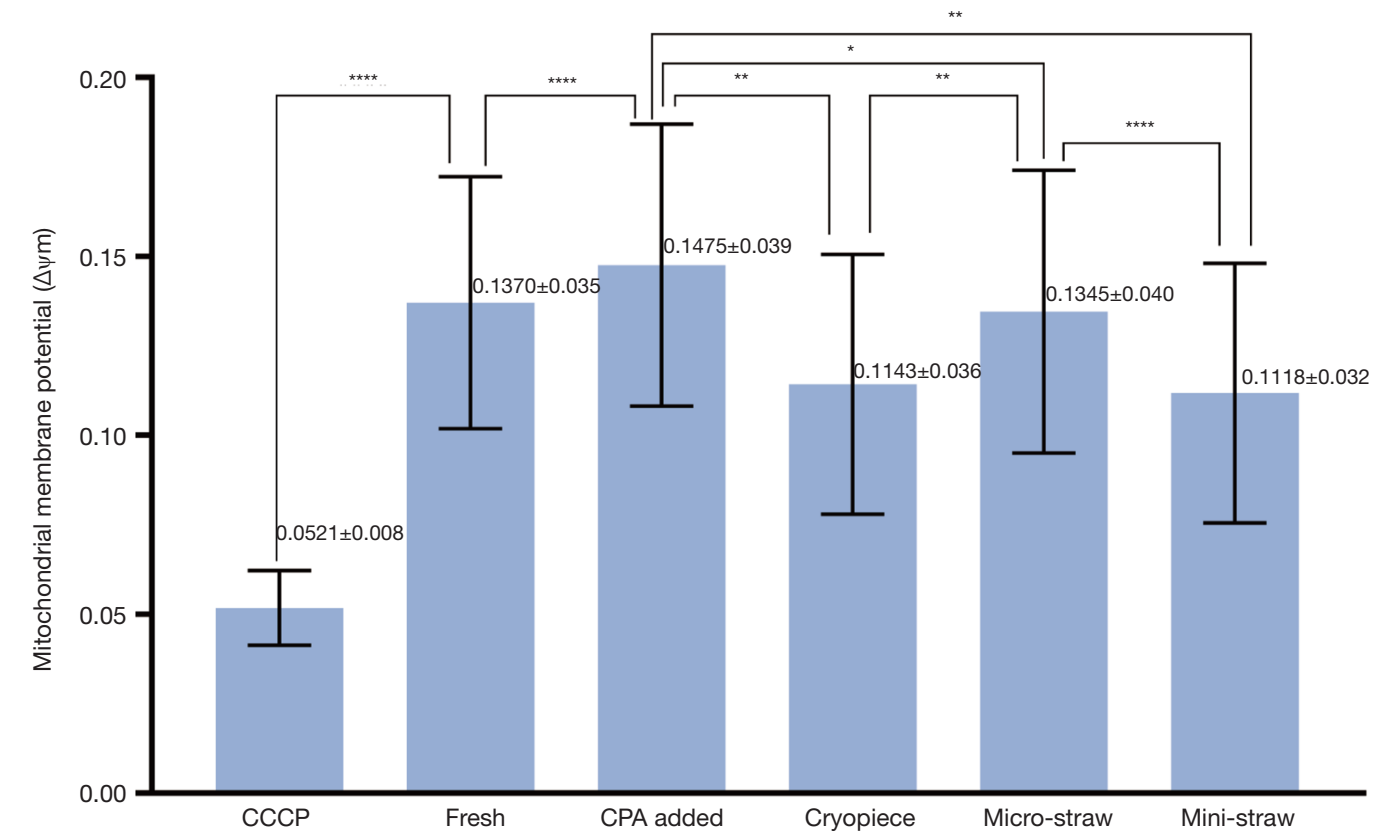

Figure 6 Flow cytometric evaluation of mitochondrial membrane potential (MMP). (A) Results of flow cytometry analysis. Red fluorescence corresponds to high $\Delta \psi \mathrm{m}$ (active and functional mitochondria), and green fluorescence corresponds to low $\Delta \psi \mathrm{m}$ (inactive or inoperative mitochondria). The red/green ratio indicates the whole mitochondrial activity in sperm; (B) comparison of sperm MMP between different groups. Though MMP of sperm changed significantly after cryopreservation, micro-straw maintained sperm MMP better than Cryopiece and mini-straw did, while no significant difference was observed in MMP between sperm cryopreserved using Cryopiece and mini-straw $\left(^{*}\right.$, $\left.\mathrm{P}<0.05 ;{ }^{* *}, \mathrm{P}<0.01 ;{ }^{* * * *}, \mathrm{P}<0.0001\right)$. CPA, cryoprotective agent. 
Sperm motility is the major factor in determining male fertility which is associated with the function of mitochondria. The most notable aspect of mitochondria function of sperm is the production of chemical energy in the form of ATP. Sperm use this energy to sustain motility. Though the sperm cryopreserved using micro-straw has the highest MMP, no difference between the MMP of the sperm cryopreserved using Cryopiece and mini-straw was observed. It was also noticed that the adding of CPA may help increasing the MMP of sperm before freezing, it was reported that the glucose in CPA could induce hyperpolarization in sperm through $\mathrm{Na}^{+}, \mathrm{K}^{+}$-ATPase (22).

Recently, a cryoprotectant-free vitrification method was introduced for the freezing of small volume of spermatozoa (23). Compared with conventional freezing methods, it provided better recovery rate and MMP. Though no retrieval rate after freezing-thawing was provided, the cryoprotectant-free vitrification method provided a cheaper and simpler choice for rare sperm cryopreservation. We'd like to try this method to improve our protocol for sperm cryopreservation. The combination of cryoprotectant-free vitrification method and Cryopiece might provide more available sperm for the patient at the day of oocyte retrieve.

In conclusion, the recovery rate and retrieval rate of the sperm cryopreserved using Cryopiece are significantly higher than that of the sperm cryopreserved using ministraw and micro-straw. Using of Cryopiece, as well as micro- or mini-straw, had no significantly impact on sperm morphology and DFI. Micro-straw maintained MMP best during cryopreservation, while no significant difference was observed in MMP between sperm cryopreserved using Cryopiece and mini-straw. The main shortcoming of our work is that sperm specimens provided from fertile donors were used instead of that derived from the patients who were the potential target users of the carriers evaluated. We did so to avoid the waste of precious, extremely rare spermatozoa retrieved from these patients, since the use of these spermatozoa in ART treatment may be the last chance for them to father their own child. Besides, the sperm retrieved from these patients are usually too few to meet the need for tests, such as the detection of DFI, morphology, and MMP, which require a large amount of sperm for detecting after treatment. Anyhow, our clinical data proved that the use of this novel carrier was beneficial for improving the outcome in the ART cycles of patients suffering from extremely severe oligospermia (17). Cryopiece is valuable for clinical practice.

\section{Acknowledgments}

We appreciate Prof. De-Yi Liu (Department of Obstetrics \& Gynecology, University of Melbourne, Australia) for language editing.

Funding: This work was supported by National Key Research and Development Program of China (2017YFC1002003), Key Research and Development Project of Ningxia (2020BFH02002), National Natural Science Foundation of China (31660308), and Interdisciplinary Program of Shanghai Jiao Tong University (YG2017ZD04).

\section{Footnote}

Reporting Checklist: The authors have completed the TREND reporting checklist. Available at http://dx.doi. org/10.21037/tau-20-1080

Data Sharing Statement: Available at http://dx.doi. org/10.21037/tau-20-1080

Peer Review File: Available at http://dx.doi.org/10.21037/ tau-20-1080

Conflicts of Interest: All authors have completed the ICMJE uniform disclosure form (available at http://dx.doi. org/10.21037/tau-20-1080). The authors have no conflicts of interest to declare.

Ethical Statement: The authors are accountable for all aspects of the work in ensuring that questions related to the accuracy or integrity of any part of the work are appropriately investigated and resolved. The study was conducted in accordance with the Declaration of Helsinki (as revised in 2013). This study was approved by the Institutional Review Board of Shanghai General Hospital (license number of ethics statement: 2016KY196). Informed consents were signed by all sperm donors.

Open Access Statement: This is an Open Access article distributed in accordance with the Creative Commons Attribution-NonCommercial-NoDerivs 4.0 International License (CC BY-NC-ND 4.0), which permits the noncommercial replication and distribution of the article with the strict proviso that no changes or edits are made and the original work is properly cited (including links to both the 
formal publication through the relevant DOI and the license). See: https://creativecommons.org/licenses/by-nc-nd/4.0/.

\section{References}

1. Mascarenhas MN, Flaxman SR, Boerma T, et al. National, regional, and global trends in infertility prevalence since 1990: a systematic analysis of 277 health surveys. PLoS Med 2012;9:e1001356.

2. Nachtigall RD. Intracytoplasmic sperm injection. West J Med 1996;165:373.

3. Palermo GD, Cohen J, Alikani M, et al. Intracytoplasmic sperm injection: a novel treatment for all forms of male factor infertility. Fertil Steril 1995;63:1231-40.

4. Mantikou E, Youssef MAFM, Wely MV, et al. Embryo culture media and IVF/ICSI success rates: a systematic review. Hum Reprod Update 2013;19:210-20.

5. Bernie AM, Ramasamy R, Stember DS, et al. Microsurgical epididymal sperm aspiration: indications, techniques and outcomes. Asian J Androl 2013;15:40-3.

6. O'Connell M, Mcclure N, Lewis SEM. The effects of cryopreservation on sperm morphology, motility and mitochondrial function. Hum Reprod 2002;17:704-9.

7. Liu F, Zou SS, Zhu Y, et al. A novel micro-straw for cryopreservation of small number of human spermatozoon. Asian J Androl 2017;19:326-9.

8. Desai NN, Blackmon H, Goldfarb J. Single sperm cryopreservation on cryoloops: an alternative to hamster zona for freezing individual sperm. Reprod Biomed Online 2004;9:47-53.

9. Chen Y, Li L, Qian Y, et al. Small-volume vitrification for human sperm in the absence of CPAs by using Cryotop. Andrologia 2015;47:694-9.

10. Coetzee K, Ozgur K, Berkkanoglu M, et al. Reliable single sperm cryopreservation in Cell Sleepers for azoospermia management. Andrologia 2016;48:203-10.

11. Endo Y, Fujii Y, Kurotsuchi S, et al. Successful delivery derived from vitrified-warmed sperm from a patient with nonobstructive azoospermia. Fertil Steril 2012;98:1423-7.

12. Endo Y, Fujii Y, Shintani K, et al. Simple vitrification for small numbers of human sperm. Reprod Biomed Online 2012;24:301-7.

13. Cohen J, Garrisi GJ, Congedo-Ferrara TA, et al. Cryopreservation of single human sperm. Hum Reprod 1997;12:994-1001.

14. Tomita K, Sakai S, Khanmohammadi M, et al.
Cryopreservation of a small number of human sperm using enzymatically fabricated, hollow hyaluronan microcapsules handled by conventional ICSI procedures. J Assist Reprod Genet 2016;33:501-11.

15. Araki Y, Yao T, Asayama Y, et al. Single human sperm cryopreservation method using hollow-core agarose capsules. Fertil Steril 2015;104:1004-9.

16. Hatakeyama S, Tokuoka S, Abe H, et al. Cryopreservation of very low numbers of sperm from male patients undergoing infertility treatment using agarose capsules. Human Cell 2017;30:201-8.

17. Sun J, Chen W, Zhou L, et al. Successful delivery derived from cryopreserved rare human sperm with novel Cryopiece. Andrology 2017;5:832-7.

18. Rahiminia T, Hosseini A, Anvari M, et al. Modern human sperm freezing:Effect on DNA, chromatin and acrosome integrity. Taiwan J Obstet Gynecol 2017;56:472-6.

19. Liu T, Gao J, Zhou N, et al. The effect of two cryopreservation methods on human sperm DNA damage. Cryobiology 2016;72:210-5.

20. Zeke J, Konc J, Kanyo K, et al. Birth and clinical pregnancy from fresh and frozen oocytes fertilized with cryopreserved testicular sperm. Syst Biol Reprod Med 2012;58:165-7.

21. Liow SL, Foong LC, Chen NQ, et al. Live birth from vitrified-warmed human oocytes fertilized with frozenthawed testicular sperm. Reprod Biomed Online 2009;19:198-201.

22. Guzmán-Grenfell AM, Bonilla-Hernández MA, GonzálezMartínez MT. Glucose induces a Na+, K+-ATPasedependent transient hyperpolarization in human sperm. I. Induction of changes in plasma membrane potential by the proton ionophore CCCP. Biochim Biophys Acta 2000;1464:188-98.

23. Spis E, Bushkovskaia A, Isachenko E, et al. Conventional freezing vs. cryoprotectant-free vitrification of epididymal (MESA) and testicular (TESE) spermatozoa: Three live births. Cryobiology 2019;90:100-102.

Cite this article as: Zhu ZJ, Zhai J, Hu JL, Wang YZ, Chen W, Liu F, Huang YH, Li Z. Cryopiece, a novel carrier with faster cooling rate, high recovery rate and retrieval rate, for individual sperm cryopreservation. Transl Androl Urol 2021;10(3):11211132. doi: $10.21037 /$ tau-20-1080 